PAPER

\title{
Lesional location of lateral medullary infarction presenting hiccups (singultus)
}

\author{
M H Park, B J Kim, S B Koh, M K Park, K W Park, D H Lee
}

See end of article for

authors' affiliations

J Neurol Neurosurg Psychiatry 2005;76:95-98. doi: 10.1136/jnnp.2004.039362

Correspondence to:

Dr Dae Hie Lee

Department of Neurology,

Korea University Medical

College, 126-1, Anam-

dong-5-ga, Sungbuk-gu,

Seoul 136-705, Republic

of Korea;

www@medimail.co.kr

Received 16February 2004

In revised form

30 March 2004

Accepted 24 April 2004
Background: Hiccups are an infrequent result of lateral medullary infarction. Their importance may be underestimated and they can cause distress, exhaustion, and aspiration. Hiccups in lateral medullary infarction remain poorly understood

Objective: To evaluate the relation between the lesional loci of lateral medullary infarction and hiccups. Methods: 51 patients with lateral medullary infarction were investigated by magnetic resonance imaging within three days of the onset of infarction. Seven of the 51 patients developed hiccup.

Results: All patients with hiccups had middle level lateral medullary lesions, including two with lower level lesions and four with upper level lesions. In the middle level lateral medullary lesions, dorsolateral lesions were most often involved. All patients with lateral medullary infarction presenting with hiccups also had vertigo, dizziness, nausea, vomiting, and dysphagia.

Conclusions: The observations suggest that middle level and dorsolateral lesion locations in lateral medullary infarction frequently induce hiccups.
L ateral medullary infarction (LMI) or Wallenberg's syndrome is one of the best known vascular syndromes of the - posterior circulation, along with events such as occlusion of the posterior inferior cerebellar artery or the vertebral artery. The usual symptoms of LMI include vertigo, dizziness, nystagmus, ataxia, nausea and vomiting, dysphagia, hoarseness, hiccups, impaired sensation over half the face, impairment of pain and thermal sensation over the contralateral hemibody and limbs and the ipsilateral face, and Horner's syndrome. ${ }^{1-3}$

Among the symptoms, hiccups (medical term, singultus) can easily be overlooked, though among other effects they may cause aspiration pneumonia, respiratory depression, and oesophagitis. ${ }^{4}$ The aetiology of hiccups includes failure of the digestive organs, irritation of the diaphragm, ingestion of alcohol, excessive smoking, and any disease of the central nervous system that involves the brain stem..$^{5-7}$ LMI in particular can be accompanied by hiccups. Unlike other symptoms and signs of LMI, however, the anatomical lesion of hiccups is not well known. ${ }^{3}$

In this study we investigated anatomical lesions in patients with LMI suffering from hiccups, using brain magnetic resonance imaging (MRI).

\section{METHODS}

\section{Subjects}

We investigated 51 patients with LMI admitted to our department of neurology between 1997 and 2002. All patients underwent MRI, which identified a medullary lesion compatible with the clinical symptoms and signs of LMI. They also had plain $x$ rays of the chest and abdomen and general blood testing. Neurological evaluation sought symptoms and signs of LMI such as vertigo, dizziness, nystagmus, ataxia, nausea and vomiting, dysphagia, hoarseness, hiccups, Horner's syndrome, impaired sensation over half the face, and impaired sensation over the hemibody and limbs.

For the evaluation of neuroanatomical lesions, we excluded any patients who might have had other causes of hiccup, such as gastrointestinal disease, irritation of the diaphragm, mediastinal or cervical disease, other CNS disease, metabolic disorders, drug treatment, and psychogenic causes. ${ }^{5-7}$ Among LMI patients with hiccups, eight who had other possible causes were excluded: two who had chronic gastritis with gastric distension, one who smoked excessively, and five who had been intubated (intubation may stimulate the glottis).

Where a nasogastric tube had been inserted, we only included patients who had hiccups before the tube was inserted and continued to have hiccups for more than 12 hours after it was removed.

After these exclusions, only seven patients were chosen for the study (five male, two female), age range 40 to 80 years, mean (SD) age, 58.14 (16.17) years.

\section{Neurological lesion}

All patients had an MRI scan, which was done on a 1.5-Tesla scanner (Vision Plus, Siemens Magnetom, Erlangen, Germany), within three days from the time of admission to hospital. Images were obtained using the fast-spin-echo method. The imaging protocol involved Tl weighted (time of repetition (TR) $570 \mathrm{~ms}$, time of echo (TE) $20 \mathrm{~ms}$ ) and T2 weighted (TR $4200 \mathrm{~ms}$, TE $96 \mathrm{~ms}$ ) transverse scan images in the horizontal plane at 5 or $6 \mathrm{~mm}$ intervals. Three axial sections of the medulla were identified as the lower, middle, and upper levels based on a study by Bradley. ${ }^{8}$ Axial cuts were further classified into intralevel lesion loci on the basis of a study by Kim et al. ${ }^{\text {T }}$ The lower level was set at the termination of the fourth ventricle, and was divided into paramedian (PM), inferior-dorsal (ID), and inferior-dorsolateral (IDL) regions. The middle level was set at the centre of the inferior olivary nucleus, and was classified into PM, midlateral (ML), and dorsolateral (DL) regions. The upper level was set at the pontobulbar junction and was split into $\mathrm{PM}$ and ML.

\section{RESULTS}

As shown in fig 1, among LMI patients with hiccup, two cases had lesion loci at the middle and lower levels, and four

Abbreviations: DL, dorsolateral; GCC, glottis closure complex; IC, inspiratory complex; ID, inferior-dorsal; IDL, inferior-dorsolateral; LMI, lateral medullary infarction; ML, midlateral; PM, paramedian 
cases at the middle and upper levels. Although one case (patient 6) had lesions over all levels of the medulla, we included this case in the analysis because the lesions were mainly located in the middle level. The lesions in the three cases involving the lower level of the medulla were all in the IDL region. Among the middle level lesions, six were in the DL region, one in the PM region, and one in the ML region. The lesions in the upper level all occurred in the ML region.

All seven subjects showed accompanying symptoms such as vertigo, dizziness, nausea/vomiting, and dysphagia. Ataxia was observed in six subjects; five experienced hoarseness, impaired sensation over half the face, and impaired sensation over the body, while three had Horner's syndrome. One subject had nystagmus. Based on the structural involvement in LMI, ${ }^{13}$ five of the seven cases had involvement the fifth nerve, with, for example, impaired sensation over half the face; six of the seven had involvement of the restiform body and cerebellar connections, with ataxia; variable numbers had involvement of the vestibular nuclei and connections, manifesting as vertigo, nausea and vomiting, and nystagmus; three of the seven cases had involvement of the descending sympathetic tract, with Horner's syndrome; and all cases had involvement of either the ninth or the tenth cranial nerves or both, with dysphagia and hoarseness.

\section{DISCUSSION}

Hiccups are repeated involuntary, spasmodic, and temporary contractions of the diaphragm accompanied by sudden closure of the glottis, producing a distinguishing "hic" sound..$^{5}$ It is suggested that hiccups are caused by the failure of the reciprocal inhibition of the valve control of the pharynx and larynx. ${ }^{10}$ This dynamic valve function is achieved by alternating excitation-inhibition of two structures: the glottis closure complex (GCC) and the inspiratory complex (IC). Activation of the GCC consists of the backward swing of the epiglottis of the vocal cords, contraction of the hyoid muscles and the superior constrictor of the pharynx, relaxation of the upper oesophageal sphincter, and activation of the IC, made up of diaphragmatic, exterior intercostal, sternocleidomastoid, anterior serratal, and scalene contraction. The IC is activated within 30-40 ms. Paradoxical activation of the GCC causes tonic phasic inspiratory obstruction. The hiccup, with its unique sound, then occurs.

The physiological purpose of hiccups is uncertain. Because fetuses and premature infants hiccup often, it is suggested that hiccups are a programmed isometric inspiratory muscle exercise, which is useless after the neonatal period but may be restimulated by irritation along the reflex arc. ${ }^{11}$

The neuroanatomical centre for hiccups is not well known, although the central connection is presumed to be a part of the brain stem which probably interacts with its respiratory centres, phrenic nerve nuclei, medullary reticular formation, and hypothalamus. ${ }^{3}{ }^{12}$ The afferent pathway is made up of the sensory branches of the phrenic and vagus nerves and the dorsal sympathetic fibres, while the efferent pathway is formed by the motor fibres of the phrenic nerve. ${ }^{13}$

Hiccups have central and peripheral causes. ${ }^{5}{ }^{14}$ Central hiccups occur with lesions between the pathway from the central nervous system to the phrenic nerve, mainly in diseases of the brain stem such as ischaemic stroke, dolichoectatic basilar artery, tumours, encephalitis, and multiple sclerosis. ${ }^{12131516}$ The role of the central nervous system is suggested by the complex physiological mechanism of hiccup, which requires an interaction in the supraspinal polysynapsis. ${ }^{13}$ Peripheral hiccups can be caused by diseases at phrenic nerve level such as gastric distension. ${ }^{5}{ }^{6}$

We cannot identify the mechanism of hiccup in patients with LMI, but we can make a few assumptions. First, it is likely that there is denervation supersensitivity because palatal myoclonus is common in such patients. ${ }^{14}{ }^{15}{ }^{17}$ Loss of supranuclear control occurs in the brain stem, especially at ponto-medullary level-the so called myoclonic triangle of Guillan-Mollaret consisting of the dentate nucleus, the inferior olivary nucleus, and the red nucleus. Denervation supersensitivity leads to hiccup. Nonetheless, the region of the myoclonic triangle of Guillan-Mollaret usually involves the peduncle rather than the medulla. Hiccups occur several weeks after the onset of denervation, when enlargement of the inferior olivary nucleus is observed. The patients in our study, however, developed hiccups on the day of the brain infarction or on the next day, and brain MRI did not show any particular lesion in the Guillan-Mollaret triangle. This mechanism was therefore not able to explain hiccups in lateral medullary infarction.

Hiccups occur because LMI causes an imbalance between expiration and inspiration. ${ }^{118}$ The expiratory centre and the inspiratory centre are in the central medulla (fig 2). The areas involved are known to overlap, the expiratory area being larger than the inspiratory area and extending more laterally and dorsally in the reticular formation of the medulla. The expiratory centre is also larger, and lies in the outer side of the medulla. Thus, if the lesion is around the nucleus ambiguus of the medulla, the expiratory centre is destroyed,

\begin{tabular}{|c|c|c|c|c|c|}
\hline $\begin{array}{l}\text { Patient } \\
\text { No/sex/age }\end{array}$ & $\begin{array}{l}\text { Level of } \\
\text { lesion }\end{array}$ & Side $^{\dagger} /$ Loci $^{\ddagger}$ of lesion & MRI findings & $\begin{array}{l}\text { TOther neurological } \\
\text { symptoms and signs }\end{array}$ & $\begin{array}{l}\text { Figure } 1 \text { Characteristics of lateral } \\
\text { medullary infarction patients with } \\
\text { hiccups. * } L \text {, lower; } M \text {, middlle; and } U \text {, }\end{array}$ \\
\hline $1 / M / 40$ & $L M$ & $R / I D L+D L$ & & $\begin{array}{l}\text { VD, NS, GA, N/V, DP, } \\
H S, H N, I F, I B\end{array}$ & $\begin{array}{l}\text { upper. †L, left; R, right. } \neq I D \text {, inferior- } \\
\text { dorsal; IDL, inferior-dorsolateral; DL, } \\
\text { dorsolateral. PM }\end{array}$ \\
\hline $2 / M / 42$ & $L M$ & $R / I D L+D L$ & & $\begin{array}{l}\text { VD, NS, GA, N/V, DP, } \\
H S, H N, I F, I B\end{array}$ & $\begin{array}{l}\text { midlateral. 'DP, dysphagia; GA, gait } \\
\text { ataxia; HN, Horner's sign; HS, }\end{array}$ \\
\hline $3 / M / 50$ & MU & $R / D L+M L$ & & $\begin{array}{l}\text { VD, GA, N/V, DP, } \\
H S, H N, I F, I B\end{array}$ & $\begin{array}{l}\text { hoarseness; IB, impaired sensation over } \\
\text { the body; IF, impaired sensation over } \\
\text { half the face; NS, nystagmus; N/V, }\end{array}$ \\
\hline $4 / M / 58$ & MU & $\mathrm{L} / \mathrm{DL}+\mathrm{ML}$ & & $\begin{array}{l}\text { VD, GA, N/V, DP, } \\
H S, I F, I B\end{array}$ & $\begin{array}{l}\text { nausea/vomiting; VD, vertigo/ } \\
\text { dizziness. }\end{array}$ \\
\hline $5 / M / 58$ & $M U$ & $\mathrm{R} / \mathrm{ML}+\mathrm{ML}$ & & VD, GA, N/V, DP, IF & \\
\hline 6/F/79 & LMU & $R / I D L+(P M+D L)+M L$ & & VD, GA, N/V, DP, IF, IB & \\
\hline $7 / F / 80$ & MU & $R / D L+D L$ & & $\mathrm{VD}, \mathrm{N} / \mathrm{V}, \mathrm{DP}, \mathrm{HS}$ & \\
\hline
\end{tabular}




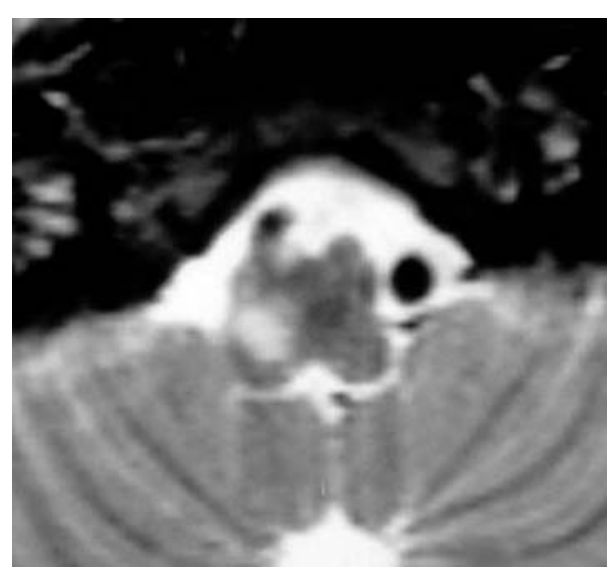

Figure 2 Major lesions of lateral medullary infarction in patients with hiccups on T2WI magnetic resonance imaging.

causing an imbalance between expiration and inspiration and consequently leading to hiccups.

In addition, hiccups may stem from ischaemia of the dorsal motor nucleus of the vagus or the nucleus of the solitary tract located beneath the lateral part of the floor of the fourth ventricle in the dorsal medulla, medial to the vestibular nuclei. ${ }^{2}$ As well as being caused by LMI, hiccups reportedly occur because of angiomas in the medulla, ${ }^{5}$ primary medullary haemorrhage, ${ }^{19}$ and tumours or tuberculomata in the brain stem. ${ }^{15}$ These are all located in the dorsolateral medulla. Five cases in our study occurred in the dorsolateral medulla. In animal experiments,$^{20}$ hiccup-like responses can be generated by electrical stimulation of the medullary reticular formation lateral to the nucleus ambiguus at the rostrocaudal level, between $1 \mathrm{~mm}$ and $2.5 \mathrm{~mm}$ rostral to the obex of the fourth ventricle of a cat. This is mainly the region of the dorsolateral medulla, where the nucleus ambiguus is located-including the vagal motor neurones projecting to the larynx and the premotor neurones that control the diaphragm. ${ }^{21}{ }^{22}$ Thus lesions in this region induce hiccups, as they affect the laryngeal motor neurones which control the glottis and the premotor neurones which control the inspiratory muscles. ${ }^{23}$ If the nucleus raphé magnus-with GABAergic inhibitory cells in the medullary reticular formation of the outer region of the nucleus ambiguus-is destroyed hiccups are induced. ${ }^{23}$

The clinical features of hiccups in LMI have been described. ${ }^{124-26}$ The frequency of hiccup is around 12-36\% in this condition. Nonetheless, the frequency of LMI itself is relatively low, and most studies involved only 20 to 40 patients, with differing frequencies of LMI. Data from a previous study on $\mathrm{LMI}^{2}$ showed that 44 of 172 patients (around 26\%) developed hiccup. ${ }^{2}$ In our study, seven of 51 patients (14\%) developed hiccup.

Hiccups in LMI are often accompanied by symptoms involving the fifth, ninth, and tenth cranial nerves, with nausea and vomiting. ${ }^{1}$ In the present study, all the affected patients had symptoms involving either the ninth or the tenth cranial nerve or both, six involved the cerebellar connections, five had symptoms of fifth cranial nerve involvement, and all cases had symptoms caused by involvement of the vestibular nuclei and their connections (figs 2 and 3). From our analyses of symptoms and lesion locations, we thought that the hiccup centre-the possible area of involvement necessary to produce hiccups-was placed in the region of the cerebellar, vestibular, and ninth, tenth, and fifth cranial nerve nuclei, and their connections with the middle part of the medulla. The lesion appears to be mainly in the dorsolateral region of the middle medulla. One

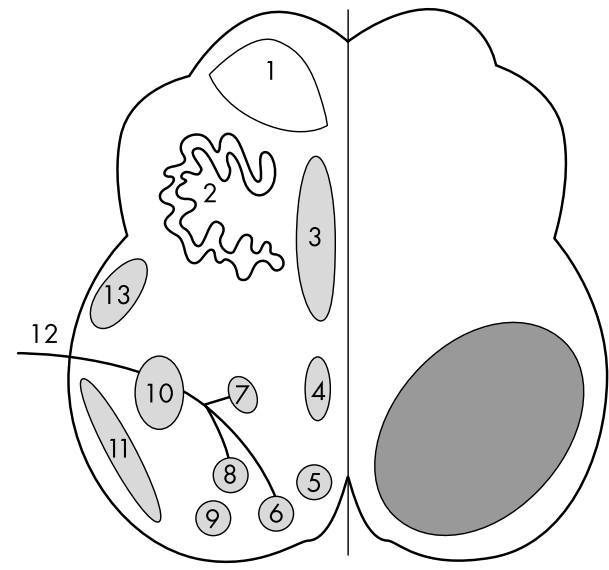

\begin{tabular}{|ll|}
\hline 1 & Pyramidal tract \\
2 & Inferior olive \\
3 & Medial lemniscus \\
4 & Medial longitudinal fasciculus \\
5 & Hypoglossal nucleus \\
6 & Dorsal nucleus of the vagus nerve \\
7 & Nucleus ambiguus \\
8 & Solitary nucleus \\
9 & Vestibular nucleus \\
10 & Spinal tract and nucleus of trigeminal nerve $(V)$ \\
11 & Posterior spinocerebellar tract \\
12 & Vagus nerve $(X)$ \\
13 & Spinothalamic tract \\
\hline
\end{tabular}

Figure 3 Schematic transverse section of the medulla. Cell columns related to functional components of important structures are indicated on the left. On the right, the hypothesised critical region involved in the development of hiccups is indicated by the dark oval.

might expect that such a lesion could also induce hiccups through an imbalance between expiration and inspiration, because it is situated around the respiratory area. This has been suggested in previous studies. ${ }^{118}$

Unfortunately, our study has some limitations. Only 1.9\% of all cerebral infarcts are in the lateral medulla, ${ }^{24}$ and our yield of only seven patients reflects this. It is therefore difficult to obtain enough patients for an adequate sample. In addition, few of the patients with LMI developed hiccup. Patients with dysphagia and secondary hiccup caused by oral secretions or irritation of the airway from dysphagia cannot easily be distinguished from those with a central cause. From previous studies, however, LMI patients experience hiccups during sleep before developing dysphagic symptoms, and the hiccups do not cease in parallel with recovery from the dysphagia. ${ }^{1}$ While MRI is the preferred imaging technique for vertebrobasilar territory infarcts, and we used an anatomical approach at three different levels and two or three intralevel areas in the medulla in order to minimise errors in locating the infarcts, there may nevertheless be sources of investigational bias; these could arise from variations in the thickness of the MRI slices and the gaps between them, and uncertainties in forming the reference line used to section the brain stem.

\section{Conclusions}

Around 14\% of patients with lateral medullary infarcts had hiccups in our study, mainly when the lesions occurred in the dorsolateral region of the middle medulla. There was a close correlation between hiccups and symptoms of cerebellar, vestibular, and fifth, ninth, and tenth cranial nerve involvement. Although there may be arguments against assigning a simple lesional location to such a complicated process as 
hiccup, the development of hiccup does appear to be related to lesions in the dorsolateral region of the middle medulla. A larger study is necessary to define the anatomical correlates of hiccup with more certainty.

\section{Authors' affiliations}

M H Park, B J Kim, S B Koh, M K Park, K W Park, D H Lee, Department of Neurology, Korea University Medical College, Seoul, Republic of Korea

Competing interests: none declared

\section{REFERENCES}

1 Currier RD, Giles CL, Dejong RN. Some comments on Wallenberg's lateral medullary syndrome. Neurology 1961;11:778-91.

2 Caplan LR. Posterior circulation disease: clinical findings, diagnosis, and management. Massachusetts: Blackwell Science, 1996:263-323.

3 Victor M, Ropper AH. Adams and Victor's principles of neurology. New York: McGraw-Hill, 2001:821-924.

4 Kumar A, Dromerick AW. Intractable hiccups during stroke rehabilitation. Arch Phys Med Rehabil 1998;79:697-9.

5 Launois S, Bizec JL, Whitelaw WA, et al. Hiccup in adults: an overview. Eur Respir J 1993;6:563-75.

6 Loft LM, Ward RF. Hiccups. A case presentation and etiologic review. Arch Otolaryngol Head Neck Surg 1992;118:1115-19.

7 Friedman NL. Hiccups: a treatment review. Pharmacotherapy 1996;16:986-95.

8 Bradley WG. MR of the brain stem: a practical approach. Radiology $1991 ; 179: 319-32$.

$9 \mathrm{Kim} \mathrm{H}$, Chung CS, Lee KH, et al. Aspiration subsequent to a pure medullary infarction: lesion sites, clinical variables, and outcome. Arch Neurol 2000;57:478-83.

10 Askenasy JJ. About the mechanism of hiccup. Eur Neurol 1992;32:159-63.
11 Kahrilas PJ, Shi G. Why do we hiccup? Gut 1997:41:712-13.

12 Marsot-Dupuch K, Bousson V, Cabane J, et al. Intractable hiccups: the role of cerebral MR in cases without systemic cause. Am J Neuroradiol 1995; 16:2093-100.

13 Fodstad H, Nilsson S. Intractable singultus: a diagnostic and therapeutic challenge. Br J Neurosurg 1993;7:255-60.

14 Musumeci A, Cristofori L, Bricolo A. Persistent hiccup as presenting symptom in medulla oblongata cavernoma: a case report and review of the literature. Clin Neurol Neurosurg 2000;102:13-17.

15 al Deeb SM, Sharif H, al Moutaery K, et al. Intractable hiccup induced by brainstem lesion. J Neurol Sci 1991;103:144-50.

16 McFarling DA, Susac JO. Hoquet diabolique: intractable hiccups as a manifestation of multiple sclerosis. Neurology 1979;29:797-801.

17 de la Fuente-Fernandez R. [Hiccup and dysfunction of the inferior olivary complex]. Med Clin (Barc) 1998; 110:22-4.

18 Hoff HE, Breckenridge CG. The neurogenesis of respiration. In: Fulton JF, ed A textbook of physiology. Philadelphia: WB Saunders Co, 1955:843-9.

19 Kumral E, Acarer A. Primary medullary haemorrhage with intractable hiccup. J Neurol 1998;245:620-2.

20 Arita $\mathrm{H}$, Oshima T, Kita l, et al. Generation of hiccup by electrical stimulation in medulla of cats. Neurosci Lett 1994;175:67-70.

21 Arita H, Sakamoto M, Hirokawa Y, et al. Serotonin innervation patterns differ among the various medullary motoneuronal groups involved in upper airway control. Exp Brain Res 1993;95:100-10.

22 Cohen MI. Neurogenesis of respiratory rhythm in the mammal. Physiol Rev 1979;59:1 105-73

23 Oshima T, Sakamoto M, Tatsuta $H$, et al. GABAergic inhibition of hiccup-like reflex induced by electrical stimulation in medulla of cats. Neurosci Res 1998;30:287-93.

24 Norrving B, Cronqvist S. Lateral medullary infarction: prognosis in an unselected series. Neurology 1991;41:244-8.

25 Sacco RL, Freddo L, Bello JA, et al. Wallenberg's lateral medullary syndrome. Clinical-magnetic resonance imaging correlations. Arch Neurol 1993;50:609-14.

26 Lee SS, Roh JK, Lee SB, et al. A clinical study of 21 patients with lateral medullary syndrome. J Korean Neurol Assoc 1989;7:42-51.

\section{Call for papers}

10th European Forum on Quality Improvement in Health Care

13-15 April 2005, ExCel, Docklands, London

For further information on how to submit your paper please go to:

http://www.quality.bmipg.com 